\title{
INFINITE MATRICES AND INVARIANT MEANS
}

\author{
PAUL SCHAEFER
}

\begin{abstract}
Let $\sigma$ be a one-to-one mapping of the set of positive integers into itself such that $\sigma^{\mathcal{D}}(n) \neq n$ for all positive integers $n$ and $p$, where $\sigma^{p}(n)=\sigma\left(\sigma^{p-1}(n)\right), p=1,2, \cdots$. A continuous linear functional $\varphi$ on the space of real bounded sequences is an invariant mean if $\varphi(x) \geqq 0$ when the sequence $x=\left\{x_{n}\right\}$ has $x_{n} \geqq 0$ for all $n$, $\varphi(\{1,1,1, \cdots\})=+1$, and $\varphi\left(\left\{x_{\sigma(n)}\right\}\right)=\varphi(x)$ for all bounded sequences $x$. Let $V_{\sigma}$ be the set of bounded sequences all of whose invariant means are equal. If $A=\left(a_{n k}\right)$ is a real infinite matrix, then $A$ is said to be (1) $\sigma$-conservative if $A x=\left\{\Sigma_{k} a_{n k} x_{k}\right\} \in V_{\sigma}$ for all convergent sequences $x$, (2) $\sigma$-regular if $A x \in V_{\sigma}$ and $\varphi(A x)=\lim x$ for all convergent sequences $x$ and all invariant means $\varphi$, and (3) $\sigma$-coercive if $A x \in V_{\sigma}$ for all bounded sequences $x$. Necessary and sufficient conditions are obtained to characterize these classes of matrices.
\end{abstract}

1. Introduction. Let $\sigma$ be a mapping of the set of positive integers into itself. A continuous linear functional $\varphi$ on $m$, the space of real bounded sequences, is said to be an invariant mean or a $\sigma$-mean if and only if (1) $\varphi(x) \geqq 0$ when the sequence $x=\left\{x_{n}\right\}$ has $x_{n} \geqq 0$ for all $n,(2) \varphi(e)=1$, where $e=\{1,1,1, \cdots\}$, and $(3) \varphi\left(\left\{x_{\sigma(n)}\right\}\right)=\varphi(x)$ for all $x \in m$. For certain kinds of mappings $\sigma$, every invariant mean $\varphi$ extends the limit functional on the space $c$ of real convergent sequences, in the sense that $\varphi(x)=\lim x$ for all $x \in c$. Consequently, $c \subset V_{\sigma}$ where $V_{\sigma}$ is the set of bounded sequences all of whose $\sigma$-means are equal.

When $\sigma(n)=n+1$, the $\sigma$-means are the classical Banach limits on $m$ and $V_{\sigma}$ is the set of almost convergent sequences [5]. If $A=\left(a_{n k}\right)$ is an infinite matrix with real entries such that $A x=\left\{\sum_{k} a_{n k} x_{k}\right\}$ is an almost convergent sequence for every convergent sequence $x, A$ is said to be an almost conservative matrix [4]. When the common value of all Banach limits of $A x$ is $\lim x$ for all $x \in c$, then the almost conservative matrix $A$ is said to be almost regular. J. P. King [4] gave necessary and sufficient conditions that a matrix be almost conservative or almost regular. More

Received by the editors July 8, 1971 and, in revised form, March 7, 1972.

AMS 1970 subject classifications. Primary 40C05, 40D05.

Key words and phrases. $\sigma$-conservative matrices, $\sigma$-regular matrices, $\sigma$-coercive matrices, invariant means, almost convergence.

(c) American Mathematical Society 1972 
recently, Eizen and Laush [2] considered the class of almost coercive matrices, those for which $A x$ is almost convergent for every bounded sequence $x$. In this paper we define analogous notions of $\sigma$-conservative, $\sigma$-regular, and $\sigma$-coercive matrices and obtain conditions which characterize them.

2. Preliminaries. We consider the spaces $c$ and $m$ as Banach spaces normed by $\|x\|=\sup \left\{\left|x_{n}\right|\right\}$. Let $c^{\prime}$ and $m^{\prime}$ denote the conjugate spaces of $c$ and $m$ respectively, normed in the usual way. It is well known that each $f \in c^{\prime}$ has the representation

$$
f(x)=(\lim x)\left[f(e)-\sum_{k=1}^{\infty} f\left(e^{k}\right)\right]+\sum_{k=1}^{\infty} x_{k} f\left(e^{k}\right),
$$

where $x=\left\{x_{k}\right\}$ and $e^{k}$ is the sequence having +1 in its $k$ th entry and zeros elsewhere. Furthermore, $\|f\|$ is given by $\left|f(e)-\sum_{k=1}^{\infty} f\left(e^{k}\right)\right|+\sum_{k=1}^{\infty}\left|f\left(e^{k}\right)\right|$. The set $\left\{e, e^{1}, e^{2}, \cdots\right\}$ is a Schauder basis for $c$, and every $x=\left\{x_{k}\right\} \in c$ can be written uniquely as $x=(\lim x) e+\sum_{k}\left(x_{k}-\lim x\right) e^{k}[8]$.

Throughout this paper we deal only with mappings $\sigma$ of the set of positive integers into itself which are one-to-one and are such that $\sigma^{p}(n) \neq n$ for all positive integers $n$ and $p$, where $\sigma^{p}(n)$ denotes the $p$ th iterate of the mapping $\sigma$ at $n$. For such mappings, every $\sigma$-mean extends the limit functional on $c[6]$.

If $x=\left\{x_{n}\right\}$, set $T x=\left\{x_{\sigma(n)}\right\}$. It can be shown that the set $V_{\sigma}$ described in the Introduction can be characterized as the set of all bounded sequences $x$ for which $\lim _{p}\left(x+T x+\cdots+T^{p} x\right) /(p+1)$ exists in the space $m$ and has the form $L e, L$ being the common value of all $\sigma$-means at $x$ [6]. We write $L=\sigma$-lim $x$.

3. $\sigma$-conservative and $\sigma$-regular matrices. All matrices in this paper are real infinite matrices. For such matrices, the notions of being almost conservative and almost regular can be generalized as follows.

Definition 1. An infinite matrix $A$ is said to be $\sigma$-conservative if and only if $A x=\left\{\sum_{k} a_{n k} x_{k}\right\} \in V_{\sigma}$ for all $x \in c$.

Definition 2. An infinite matrix $A$ is said to be $\sigma$-regular if and only if it is $\sigma$-conservative and $\sigma-\lim A x=\lim x$ for all $x \in c$.

THEOREM 1. The matrix $A$ is $\sigma$-conservative if and only if

(1) $\|A\|=\sup _{n}\left\{\sum_{k}\left|a_{n k}\right|\right\}<+\infty$,

(2) $a_{(k)}=\left\{a_{n k}\right\}_{n=1}^{\infty} \in V_{\sigma}$ for each $k$, and

(3) $a=\left\{\sum_{k} a_{n k}\right\}_{n=1}^{\infty} \in V_{\sigma}$.

When $A$ is $\sigma$-conservative, the $\sigma$-limit of $A x$ is $(\lim x)\left[u-\sum_{k} u_{k}\right]+\sum_{k} x_{k} u_{k}$ for every $x=\left\{x_{k}\right\} \in c$, where $u=\sigma-\lim a$ and $u_{k}=\sigma-\lim a_{(k)}, k=1,2, \cdots$. 
THEOREM 2. The matrix $A$ is $\sigma$-regular if and only if

(1) $\|A\|<+\infty$,

(2) $a_{(k)} \in V_{\sigma}$ with $\sigma$-limit zero for each $k$, and

(3) $a \in V_{\sigma}$ with $\sigma$-limit +1 .

For typographical convenience we shall use the notation $a(n, k)$ to denote the element $a_{n k}$ of the matrix $A$ in the following proofs.

Proof of Theorem 1. Let us first suppose that conditions (1), (2) and (3) hold. Let $p$ be any nonnegative integer and let $x \in c$. We have

$$
\begin{aligned}
& \left(A x+T A x+\cdots+T^{p} A x\right) /(p+1) \\
& \quad=\left\{\sum_{k}\left[a(n, k)+a(\sigma(n), k)+\cdots+a\left(\sigma^{p}(n), k\right)\right] x_{k} /(p+1)\right\}_{n=1}^{\infty} .
\end{aligned}
$$

For every positive integer $n$, set

Then we have

$$
t_{p n}(x)=\sum_{k=1}^{\infty} \sum_{j=0}^{n} a\left(\sigma^{j}(n), k\right) x_{k} /(p+1) .
$$

$$
\begin{aligned}
\left|t_{p n}(x)\right| & \leqq \sum_{k=1}^{\infty} \sum_{j=0}^{p}\left|a\left(\sigma^{j}(n), k\right)\right| \cdot\left|x_{k}\right| /(p+1) \\
& \leqq[\|x\| /(p+1)] \cdot\left[\sum_{j=0}^{p} \sum_{k=1}^{\infty}\left|a\left(\sigma^{j}(n), k\right)\right|\right] \leqq\|A\| \cdot\|x\| .
\end{aligned}
$$

Since $t_{p n}$ is obviously linear on $c$, it follows that $t_{p n} \in c^{\prime}$ and that $\left\|t_{p n}\right\| \leqq\|A\|$.

Now,

$$
t_{p n}(e)=\left[\sum_{k=1}^{\infty} \sum_{j=0}^{p} a\left(\sigma^{j}(n), k\right)\right] /(p+1)=\left[\sum_{j=0}^{n} \sum_{k=1}^{\infty} a\left(\sigma^{j}(n), k\right)\right] /(p+1),
$$

so $\lim _{p} t_{p n}(e)$ exists uniformly in $n$ and equals $u$, the $\sigma$-limit of $a$, since $a \in V_{\sigma}$. Similarly, $\lim _{p} t_{p n}\left(e^{k}\right)=u_{k}$, the $\sigma$-limit of $a_{(k)}$ for each $k$, uniformly in $n$. Since $\left\{e, e^{1}, e^{2}, \cdots\right\}$ is a fundamental set in $c$, and $\sup _{p}\left\{\left|t_{p n}(x)\right|\right\}$ is finite for each $x \in c$, it follows that $\lim _{p} t_{p n}(x)=t_{n}(x)$ exists for all $x \in c$ $\left[1\right.$, p. 60]. Furthermore, $\left\|t_{n}\right\| \leqq \lim \inf _{p}\left\|t_{p n}\right\| \leqq\|A\|$ for each $n$, and $t_{n} \in c^{\prime}$. Thus,

$$
\begin{aligned}
t_{n}(x) & =(\lim x)\left[t_{n}(e)-\sum_{k} t_{n}\left(e^{k}\right)\right]+\sum_{k} x_{k} t_{n}\left(e^{k}\right) \\
& =(\lim x)\left[u-\sum_{k} u_{k}\right]+\sum_{k} x_{k} u_{k},
\end{aligned}
$$

an expression independent of $n$. Denote this expression by $L(x)$. 
In order to see that $\lim _{p} t_{p n}(x)=L(x)$ uniformly in $n$, set $F_{p n}(x)=$ $t_{p n}(x)-L(x)$. Then $F_{p n} \in c^{\prime},\left\|F_{p n}\right\| \leqq 2\|A\|$ for all $p$ and $n, \lim _{p} F_{p n}(e)=0$ uniformly in $n$, and $\lim _{p} F_{p n}\left(e^{k}\right)=0$ uniformly in $n$ for each $k$. Let $K$ be an arbitrary positive integer. Then

so we have

$$
x=(\lim x) e+\sum_{k=1}^{K}\left(x_{k}-\lim x\right) e^{k}+\sum_{k=K^{-1}+1}^{\infty}\left(x_{k}-\lim x\right) e^{k},
$$

Now,

$$
\begin{aligned}
F_{p n}(x)= & (\lim x) F_{p n}(e)+\sum_{k=1}^{K}\left(x_{k}-\lim x\right) F_{p n}\left(e^{k}\right) \\
& +F_{p n}\left(\sum_{k=K+1}^{\infty}\left(x_{k}-\lim x\right) e^{k}\right) .
\end{aligned}
$$

$$
\left|F_{p n}\left(\sum_{k=K+1}^{\infty}\left(x_{k}-\lim x\right) e^{k}\right)\right| \leqq 2\|A\| \cdot \sup _{k \geqq K+1}\left\{\left|x_{k}-\lim x\right|\right\}
$$

for all $p$ and $n$. By first choosing a fixed $K$ large enough, it is easy to see that each of the three displayed terms for $F_{p n}(x)$ can be made to be uniformly small in absolute value for all sufficiently large $p$, so $\lim _{p} F_{p n}(x)=0$ uniformly in $n$. This shows that

$$
\lim _{p}\left(A x+T A x+\cdots+T^{p} A x\right) /(p+1)=L(x) e,
$$

so that $A x \in V_{\sigma}$ and the matrix $A$ is $\sigma$-conservative.

Conversely, suppose that $A$ is $\sigma$-conservative. If $x$ is any null sequence, then $A x \in V_{\sigma} \subset m$. It follows from the proof of [3, Theorem 1, pp. 45 and 46] that $\|A\|<+\infty$. Furthermore, since $A e=a$ and $A e^{k}=a_{(k)}$, the other two conditions are necessary for $\sigma$-conservative matrices.

Proof of Theorem 2. If a matrix $A$ satisfies the three conditions of the theorem, then it is a $\sigma$-conservative matrix. For $x \in c$, the $\sigma$-limit of $A x$ is $L(x)$, which reduces to $\lim x$, since $u=1$ and $u_{k}=0$ for each $k$. Hence, $A$ is a $\sigma$-regular matrix. Conversely, if $A$ is $\sigma$-regular, then $\sigma-\lim A e=+1=$ $\sigma-\lim a, \sigma-\lim A e^{k}=0=\sigma-\lim a_{(k)}$, and $\|A\|$ is finite, as in the proof of Theorem 1 .

\section{4. $\sigma$-coercive matrices.}

Definition 3. A matrix $A$ is $\sigma$-coercive if and only if $A x \in V_{\sigma}$ for all $x \in m$.

THEOREM 3. The matrix $A$ is $\sigma$-coercive if and only if

(1) $\|A\|$ is finite,

(2) $a_{(k)} \in V_{\sigma}$ for each $k$, and

(3) $\lim _{p} \sum_{k=1}^{\infty}\left|\sum_{j=0}^{p}\left[a\left(\sigma^{j}(n), k\right)-u_{k}\right]\right| /(p+1)=0$ uniformly in $n$, where 
$u_{k}=\sigma-\lim a_{(k)}$. In this case, the $\sigma$-limit of $A x$ is $\sum_{k} u_{k} x_{k}$ for every $x=$ $\left\{x_{k}\right\} \in m$.

Proof. Let us first assume that the matrix $A$ satisfies conditions (1), (2) and (3). For any positive integer $K$,

$$
\begin{aligned}
\sum_{k=1}^{K}\left|u_{k}\right| & =\sum_{k=1}^{K} \lim _{p}\left|\sum_{j=0}^{p} a\left(\sigma^{j}(n), k\right)\right| /(p+1) \\
& =\lim _{p} \sum_{k=1}^{K}\left|\sum_{j=0}^{p} a\left(\sigma^{j}(n), k\right)\right| /(p+1) \\
& \leqq \underset{p}{\lim \sup } \sum_{j=0}^{p} \sum_{k=1}^{\infty}\left|a\left(\sigma^{j}(n), k\right)\right| /(p+1) \leqq\|A\| .
\end{aligned}
$$

This shows that $\sum_{k=1}^{\infty}\left|u_{k}\right|$ converges, and that $\sum_{k} u_{k} x_{k}$ is defined for every bounded sequence $x=\left\{x_{k}\right\}$.

Let $x$ be an arbitrary bounded sequence. For every positive integer $p$,

$$
\begin{aligned}
\left(A x+T A x+\cdots+T^{p} A x\right) /(p & +1)-\left(\sum_{k} u_{k} x_{k}\right) e \\
& =\left\{\sum_{k=1}^{\infty}\left(\sum_{j=0}^{p}\left[a\left(\sigma^{j}(n), k\right)-u_{k}\right] /(p+1)\right) x_{k}\right\}
\end{aligned}
$$

SO

$$
\begin{aligned}
\|(A x+T A x+\cdots+ & \left.T^{p} A x\right) /(p+1)-\left(\sum_{k} u_{k} x_{k}\right) e \| \\
& =\sup _{n}\left\{\mid \sum_{k=1}^{\infty}\left(\sum_{j=0}^{p}\left[a\left(\sigma^{j}(n), k\right)-u_{k}\right] /(p+1)\right) x_{k} \|\right\} \\
& \leqq\|x\| \cdot \sup _{n}\left\{\sum_{k=1}^{\infty}\left|\sum_{j=0}^{p}\left[a\left(\sigma^{j}(n), k\right)-u_{k}\right]\right| /(p+1)\right\} .
\end{aligned}
$$

Let $p \rightarrow+\infty$. By the uniformity of the limits in condition (3), it follows that $\left(A x+T A x+\cdots+T^{p} A x\right) /(p+1) \rightarrow\left(\sum_{k} u_{k} x_{k}\right) e$, and that $A x \in V_{\sigma}$ with $\sigma$-limit $\sum_{k} u_{k} x_{k}$.

Next, suppose that $A$ is a $\sigma$-coercive matrix. Then, since $A$ is $\sigma$-conservative, we have conditions (1) and (2) from Theorem 1 . In order to see that (3) holds, we proceed as in [2], by first showing that the limit in question is zero for each $n$, and secondly showing that the limit is uniform in $n$.

Thus, suppose that for some $n$, we have

$$
\underset{p}{\lim \sup } \sum_{k=1}^{\infty}\left|\sum_{j=0}^{p}\left[a\left(\sigma^{j}(n), k\right)-u_{k}\right]\right| /(p+1)=N>0 .
$$


Since $\|A\|$ is finite, $N$ is finite also. We observe that since $\sum\left|u_{k}\right|<+\infty$, the matrix $B=\left(b_{n k}\right)$, where $b_{n k}=a_{n k}-u_{k}$, is also a $\sigma$-coercive matrix. If one sets $F_{k p}=\left|\sum_{j=0}^{p}\left[a\left(\sigma^{j}(n), k\right)-u_{k}\right]\right| /(p+1)$, and $E_{k t}=F_{k, p_{t}}$, one can follow the construction in the proof of Theorem 2.1 in [2] to obtain a bounded sequence whose transform by the matrix $B$ is not in $V_{\sigma}$. This contradiction shows that the limit in (3) is zero for every $n$.

To show that this convergence is uniform in $n$, we invoke the following lemma, which is proved in [7].

LEMMA. Let $\{H(n)\}$ be a countable family of matrices $H(n)=\left(h_{p k}(n)\right)$ such that $\|H(n)\| \leqq M<+\infty$ for all $n$ and $\lim _{p} h_{p k}(n)=0$ for each $k$, uniformly in $n$. Then $\lim _{p} \sum_{k} h_{p k}(n) x_{k}=0$ uniformly in $n$ for all $x \in m$ if and only if $\lim _{p} \sum_{k}\left|h_{p k}(n)\right|=0$ uniformly in $n$.

We let $h_{p k}(n)=\sum_{j=0}^{p}\left[a\left(\sigma^{j}(n), k\right)-u_{k}\right] /(p+1)$ and let $H(n)$ be the matrix $\left(h_{p k}(n)\right)$. It is easy to see that $\|H(n)\| \leqq 2\|A\|$ for every $n$, and that $\lim _{p} h_{p k}(n)=0$ for each $k$, uniformly in $n$ by condition (2). For any $x \in m$, $\lim _{p} \sum_{k} h_{p k}(n) x_{k}=\sigma-\lim A x-\sum_{k} u_{k} x_{k}$, and the limit exists uniformly in $n$ since $A x \in V_{\sigma}$. Moreover this limit is zero since

$$
\left|\sum_{k} h_{p k}(n) x_{k}\right| \leqq\|x\| \cdot \sum_{k}\left|\sum_{j=0}^{p}\left[a\left(\sigma^{j}(n), k\right)-u_{k}\right]\right| /(p+1) .
$$

Thus, $\lim _{p} \sum_{k}\left|h_{p k}(n)\right|=0$ uniformly in $n$, and the matrix $A$ satisfies condition (3).

THEOREM 4. The classes of $\sigma$-regular and- $\sigma$-coercive matrices are disjoint.

Proof. If $A$ were a $\sigma$-regular and a $\sigma$-conservative matrix, then $\sigma-\lim a_{(k)}=0=u_{k}$ for every $k$. The conditions $\sigma-\lim a=+1$ and

$$
\lim _{p} \sum_{k}\left|\sum_{j=0}^{p} a\left(\sigma^{j}(n), k\right)\right|=0
$$

are incompatible, since

$$
\begin{aligned}
\left|\frac{1}{p+1} \sum_{j=0}^{p} \sum_{k} a\left(\sigma^{j}(n), k\right)\right| & =\left|\sum_{k} \frac{1}{p+1} \sum_{j=0}^{n} a\left(\sigma^{j}(n), k\right)\right| \\
& \leqq \sum_{k} \frac{1}{p+1}\left|\sum_{j=0}^{n} a\left(\sigma^{j}(n), k\right)\right|
\end{aligned}
$$




\section{REFERENCES}

1. N. Dunford and J. T. Schwartz, Linear operators. I: General theory, Pure and Appl. Math., vol. 7, Interscience, New York, 1958. MR 22 \#8302.

2. C. Eizen and G. Laush, Infinite matrices and almost convergence, Math. Japon. 14 (1969), 137-143. MR 42 \#723.

3. G. H. Hardy, Divergent series, Clarendon Press, Oxford, 1949. MR 11, 25.

4. J. P. King, Almost summable sequences, Proc. Amer. Math. Soc. 17 (1966), 12191225. MR 34 \#1752.

5. G. G. Lorentz, A contribution to the theory of divergent sequences, Acta Math. 80 (1948), 167-190. MR 10, 367.

6. R. A. Raimi, Invariant means and invariant matrix methods of summability, Duke Math. J. 30 (1963), 81-94. MR 27 \#3965.

7. P. Schaefer, Matrix transformations of almost convergent sequences, Math. Z. 112 (1969), 321-325. MR 40 \#7674.

8. A. Wilansky, Functional analysis, Blaisdell, Waltham, Mass., 1964. MR 30 \#425.

Department of Mathematics, State University College of New York, Geneseo, NEW YORK 14454 